\title{
Preliminary Planning for Mucormycosis in Developing Countries During COVID-19 Pandemic
}

\author{
Letter to the Editor $\bullet$ Neha Shakrawal, ๑ Mahendra Chouhan, ๑ Bharti Solanki \\ Department of Otorhinolaryngology and Head-Neck Surgery, Dr. Sampurnanand Medical College, Jodhpur, \\ Rajasthan, India
}

\section{ORCID ID of the authors:}

N.S. 0000-0002-5791-1716; M.C. 0000-0002-4382-5040; B.S. 0000-0001-9209-5497.

\section{Cite this article as: Shakrawal N, Chouhan} M, Solanki B. Preliminary Planning for Mucormycosis in Developing Countries During COVID-19 Pandemic. Turk Arch Otorhinolaryngol 2021; 59(4): 302-3.

Corresponding Author: Neha Shakrawal;drnehasnmc@gmail.com

Received Date: 05.06 .2021 Accepted Date: 19.10.2021

Content of this journal is licensed under a Creative Commons Attribution 4.0 International License. Available online at www.turkarchotolaryngol.net

\section{(c) (1) ()}

D0l: 10.4274/tao.2021.2021-6-1

\section{Dear Editor,}

The coronavirus pandemic was formally declared a public health emergency of international concern (PHEIC) on $30^{\text {th }}$ January 2020. In India, an upsurge of rampant opportunistic infection of coronavirus disease-2019 (COVID-19) associated mucormycosis (CAM), along with the skewed second wave of the COVID-19, has been an issue of concern from May to July 2021. Suppressed immunity due to COVID-19 and its management has created more opportunities for invasive fungal coinfections in immunocompetent individuals.

India is said to be the world capital of both diabetes and mucormycosis and accounts for $40 \%$ of the global burden of mucormycosis (1). Diabetes mellitus is the single most common risk factor for mucormycosis in India. Various authors have reported more than $70 \%$ of cases of CAM are attributed to India $(2,3)$.

The sudden increase of mucormycosis in India specifically might be the result of an unfortunate triad of uncontrolled diabetes (high prevalence), injudicious use of corticosteroid, and COVID-19 infection.

Like other resource-constrained countries, we tried hard to overcome the shortage of protective equipment, and antivirals. Now we have reached a stage with a scarcity of antifungals. Surgical debridement of mucormycosis in the absence of adequate antifungals is a futile exercise. The decline in the surge can be seen with the combined efforts of public and healthcare professionals. Preventive measures and early detection will potentially bend the curve.

\section{Preventive measures}

1. The primary measure will be the prevention of the outspread of the COVID-19 and the promotion of vaccination against it.

2. High-risk patients with poor immunity and uncontrolled glycemic state should be advised to be extra cautious. All attempts to avoid inhalation of fungal spores through the nose should be made. 
3. Avoidance of hot humid climate, which might have high environmental spore count, air purifiers may be beneficial.

4. Judicious use of corticosteroids and antifungals should be a practice to step-down the incidence of CAM and to reduce antifungal resistance.

5. Strict glycemic control during the management of COVID-19 and after recovery is paramount.

6. Immunosuppressive drugs like monoclonal antibodies and others which target the immune system should be discouraged.

7. Swab sticks for potassium hydroxide calcofluor white mount $(\mathrm{KOH})$ should be sterile and not spread crossinfection.

\section{Measures for early detection}

1. Comprehensive examination of COVID-19 patients and detecting early warning symptoms can identify CAM at the earliest. Nasal obstruction in the form of crusting or discharge are the most common initial symptoms. Blackening of any facial part suggests fungal angioinvasion. Unilateral facial numbness and pain indicate the involvement of the trigeminal nerve. Toothache, loosening of tooth, palatal eschar, facial palsy, and headache are other characteristic findings. Ptosis, periorbital swelling, diplopia, blurred and reduced vision, proptosis indicates orbital involvement. Focal seizures indicate intracranial extension (4).

2. Diagnostic nasal endoscopy and biopsy should be a practice in high-risk patients with high inflammatory markers, on poor glycemic control, on mechanical ventilation, and who have spent more than 50 days in the $\operatorname{ICU}(5)$.

3. High-risk patients should be planned for contrast magnetic resonance imaging of paranasal sinuses, orbit, and brain.
To conclude, developing countries are vulnerable to the spread of pandemic. Stringent healthcare infrastructure and public awareness are paramount to recover from this human crisis. We are all in this together and shall evolve in a better way.

Peer-review: Externally peer-reviewed.

\section{Authorship Contributions}

Conception: N.S., M.C., B.S., Design: N.S., M.C., B.S., Analysis and/or Interpretation: N.S., M.C., B.S., Literature Review: N.S., M.C., B.S., Writing: N.S., M.C., B.S.

Conflict of Interest: No conflict of interest was declared by the authors.

Financial Disclosure: The authors declared that this study received no financial support.

\section{References}

1. Prakash H, Chakrabarti A. Global epidemiology of mucormycosis. J Fungi (Basel) 2019; 5: 26. [Crossref]

2. John TM, Jacob CN, Kontoyiannis DP. When uncontrolled diabetes mellitus and severe COVID-19 converge: the perfect storm for mucormycosis. J Fungi (Basel) 2021; 7: 298. [Crossref]

3. Pal R, Singh B, Bhadada SK, Banerjee M, Bhogal RS, Hage N, et al. COVID-19-associated mucormycosis: an updated systematic review of literature. Mycoses 2021; 64: 1452-9. [Crossref]

4. Honavar SG. Code mucor: guidelines for the diagnosis, staging and management of rhino-orbito-cerebral mucormycosis in the setting of COVID-19. Indian J Ophthalmol 2021; 69: 1361-5. [Crossref]

5. Yang X, Yu Y, Xu J, Shu H, Xia J, Liu H, et al. Clinical course and outcomes of critically ill patients with SARS-CoV-2 pneumonia in Wuhan, China: a single-centered, retrospective, observational study. Lancet Respir Med 2020; 8: 475-81. [Crossref] 